\title{
AVALIAÇÃO DOS FRAGMENTOS FLORESTAIS DO MUNICÍPIO DE JABOTICABAL-SP ${ }^{1}$
}

\author{
Thiago Claudino Greggio², Teresa Cristina Tarlé Pissarra ${ }^{3}$ e Flávia Mazzer Rodrigues ${ }^{4}$
}

\begin{abstract}
RESUMO - Este estudo foi conduzido no Município de Jaboticabal, SP, com o objetivo de avaliar a evolução temporal e o número de fragmentos florestais no período de 29 anos, utilizando-se técnicas de sensoriamento remoto e fotointerpretação. Para a elaboração dos mapas foram utilizadas Cartas do IBGE de 1971 e fotografias áreas de 2000. Os resultados apontaram diminuição das áreas de floresta. Em 1971, o município apresentava $3,63 \%$ da área total com fragmentos florestais, e em 2000 observou-se, apenas, 1,55\% dessa área. Tal fato ocorreu tendo em vista o avanço de práticas agrícolas com predominância da cultura de cana-de-açúcar. A porcentagem de fragmentos florestais em 1971, com áreas menores que 10 ha, era de 46,72\%, já em 2000 esse número passou para $78,51 \%$, concluindo-se um processo de fragmentação acentuado $(31,79 \%)$ no período analisado. Os fragmentos florestais com maior extensão em 1971 se apresentaram extremamente fragmentados em 2000. Aproximadamente $60 \%$ dos fragmentos, nas duas épocas, apresentavam forma alongada, indicando alta relação perímetro/área.
\end{abstract}

Palavras-chave: Fragmentos florestais, mapeamento e sensoriamento remoto.

\section{EVALUATION OF FOREST FRAGMENT IN JABOTICABAL-S.P.}

\begin{abstract}
This study was conducted inJaboticabal-SP, to evaluate the evolution and number of forest fragments in the period of 29 years, using remote sensing and photointerpretation techniques. The IBGE Maps (1971) and 2000 aerial photos were used to map the area. The reduction of the natural forest areas occurred during the time evaluated. In 1971, the municipality presented $3.63 \%$ of the total area in forest fragments, but only $1.55 \%$ of this area in 2000. This was due to the advance of agricultural practices, mainly sugarcane, in the area. The percentage of forest fragments lower than 10ha, in 1971, was $46.72 \%$ of the total area, but in 2000, this number increased to $78.51 \%$, reaching a marked fragmentation process (31.79\%)in the analyzed period. The forest fragments with higher extension in 1971 were extremely fragmented in 2000. Approximately $60 \%$ of these areas, at both times, presented long forms, indicating a high relation between perimeter and area.
\end{abstract}

Keywords: Forest fragment, mapping and remote sensing.

\section{INTRODUÇÃO}

Estudos de longo prazo sobre mudanças espaciais e temporais de remanescentes naturais de florestas tropicais são importantes para permitir a distinção entre processos dinâmicos naturais e mudanças resultantes da ação antrópica (KORNIG e BALSLEV, 1994). Nesses remanescentes, a interação dos fatores bióticos e abióticos com as variações das comunidades e populações da fauna e flora, ao longo do tempo, determina o processo evolutivo da comunidade florestal (APPOLINÁRIO et al., 2005).

Segundo Pulliam (1996), essas interações ambientais não são estáticas no tempo, redundando em heterogeneidade espaço-temporal que varia em função do estádio sucessional, da produtividade do habitat e da estocasticidade ambiental e demográfica.

\footnotetext{
${ }^{1}$ Recebido em 26.04.2007 e aceito para publicação em 26.01.2009.

${ }^{2}$ Programa de Pós-Graduação em Microbiologia Agropecuária da Universidade Estadual Paulista Júlio de Mesquita Filho (UNESP). E-mail: <tcgreggio@yahoo.com.br>.

${ }^{3}$ Departamento de Engenharia Rural da UNESP. E-mail: <teresap@ fcav.unesp.br>.

${ }^{4}$ Programa de Pós-Graduação em Produção Vegetal da (UNESP). E-mail: <flamazzer@ hotmail.com>.
} 
Nos últimos tempos, o que se verifica no uso da terra é uma ocupação desordenada que resulta na fragmentação dos remanescentes naturais de florestas tropicais. A fragmentação florestal é um dos fenômenos mais marcantes e graves do processo de expansão da fronteira agrícola no Brasil.

O aspecto mais grave do processo de fragmentação florestal são a perda da biodiversidade e o efeito de borda. A borda do fragmento florestal é a área por onde se inicia a maior parte dos processos físicos e biológicos ligados à fragmentação. Entre os impactos negativos desse processo, cita-se o aumento de plantas invasoras que abafa outras espécies vegetais de importância para a configuração e longevidade do fragmento. Ainda, Viana (1990) chamou a atenção para o distúrbio do regime hidrológico das bacias hidrográficas, degradação dos recursos naturais e a deterioração da biodiversidade.

Os chamados países em desenvolvimento ou do Terceiro Mundo são justamente aqueles que necessitam da aplicação de vultosas quantias em mapeamento e sistemas de informação sobre áreas degradadas, com a intenção de evitar e prevenir o desperdício dos recursos naturais.

Nesse aspecto, os mapas são elementos que permitem delinear alternativas de ações e planejamento do meio e indicar respostas às várias questões sobre planejamento regional e levantamento dos recursos renováveis. Com isso, pode-se prevenir a extinção da fauna e da flora e auxiliar o processo de tomada de decisões, no que tange à formação de corredores biológicos e preservação dos fragmentos remanescentes.

Pissarra (2002) realizou o mapeamento do uso/ ocupação do solo na microbacia hidrográfica do córrego Rico do Município de Jaboticabal, SP, e em conjunto com trabalhos em campo. Concluiu que os fragmentos florestais se encontravam em estado avançado de degradação.

Nesse sentido, vê-se a importância do estudo da evolução dos fragmentos florestais em municípios, principalmente para definir programas de manejo e locar futuros corredores biológicos para incrementar a fauna e flora não só no município, mas também no Estado e na Federação.

O objetivo deste trabalho foi avaliar, no espaço e ao longo do tempo, a forma e as áreas de remanescentes de fragmentos florestais do Município de Jaboticabal e apresentar um mapa da distribuição espacial destes, no intuito de coletar subsídios para elaborar um diagnóstico conservacionista.

\section{MATERIAL E MÉTODO}

O Município de Jaboticabal está localizado na porção Centro-Norte do Estado de São Paulo, no Planalto Ocidental Paulista, tendo como referência as coordenadas geográficas $21^{\circ} 15^{\prime} 22^{\prime \prime}$ Latitude Sul e 48 $18^{\circ}$ ' $58^{\prime \prime}$ Longitude Oeste de Greenwich. Apresenta área territorial de aproximadamente $708,6 \mathrm{~km}^{2}$, dividida entre as Sedes de Jaboticabal, Distrito de Córrego Rico e Distrito Lusitânia, com $36,0 \mathrm{~km}^{2}, 2,4 \mathrm{~km}^{2}$ e $0,8 \mathrm{~km}^{2}$, respectivamente, e área rural de 669,4 km² (IBGE, 2000).

Segundo a classificação de Köppen, o clima é do tipo Cwa- subtropical-mesotérmico. A temperatura média anual na região é de $22,0^{\circ} \mathrm{C}$, a precipitação média anual está ao redor de 1.424,6 mm. Os solos predominantes são Latossolo Vermelho-Escuro de fase arenosa e Latossolo Roxo. O relevo apresenta-se em grande extensão como suave-ondulado e ondulado, com altitude variando entre 465 e 685 m (IBGE, 1971).

A floresta latifoliada tropical e trechos de Cerrado predominavam como cobertura vegetal. Com o avanço da monocultura, inicialmente pelo café e, posteriormente, por citrus e cana de açúcar, houve excessivo desmatamento, restando poucas reservas de mata nativa (Jaboticabal, 2006).

O Município de Jaboticabal está vinculado ao Comitê de Bacias do Rio Mogi-Guaçu (CBH-Mogi), segundo a Divisão Hidrográfica do Estado de São Paulo (SÃO PAULO, 1993), e através de suas inúmeras microbacias drena $100 \%$ de suas águas em redes de drenagem que deságuam no rio Mogi-Guaçu.

No estudo da rede de drenagem e localização dos fragmentos florestais do Município de Jaboticabal foram utilizadas fotografias aéreas verticais, coloridas, pertencentes à cobertura aerofotogramétrica de 2000, realizada pela BASE-Aerolevantamento, em escala nominal aproximada 1:30.000, com recobrimento longitudinal de aproximadamente $60 \%$ na mesma faixa de voo e $30 \%$ entre faixas adjacentes e Cartas do Brasil, em escala 1:50.000, editadas em 1971, pelo Instituto Brasileiro de Geografia e Estatística - IBGE, Folhas da Região de Jaboticabal (SF-22-X-D-III-3), Taquaritinga (SF-22-X-D-II-4), Guariba (SF-22-X-D-III-4), Piranji (SF-22-X-D-II-4), Taiuva (SF22-X-D-III-1) e de Pitangueiras (SF-22-X-D-III-2). 
Para a observação estereoscópica dos pares de fotografias aéreas, foi utilizado estereoscópio de espelho marca Wild, modelo ST4, decalcando-se em templetes a delimitação dos fragmentos florestais manualmente, utilizando primeiramente a demarcação das rede de drenagem e, em seguida, as linhas do contorno de cada fragmento florestal. O mapa foi elaborado em papelpoliéster, Terkron D 50 microns, e após a elaboração do Mapa Base da rede de drenagem e fragmentos florestais, o mesmo copiado e vetorizado. Para a importação do mapa no programa informatizado, foi realizada a georreferência em coordenadas dos quatro vértices da área e cinco pontos de controle. Cada feição (fragmento florestal) de interesse foi identificada em uma sequência numérica e, em seguida, determinada a sua localização e área. Após a vetorização foi efetuada a verificação dos erros e edição no próprio programa. A comparação visual das áreas de cada fragmento entre o mapa digital elaborado a partir das fotografias aéreas e o mapa digital da Carta do Brasil foi realizada no Programa AutoCad, com ajuste da escala 1:30.000, objetivando a correção de possíveis interpretações conflitantes. Para o tratamento e análise dos dados levantados, foram utilizados programas informatizados de desenho (AutoCad) e estatísticos (SAS).

O índice de circularidade ou da relação borda/ interior foi determinado para propor a tendência em relação à forma de um fragmento, conforme se segue (CHATURVEDI (1926), citado por Oliveira et. al., 2005.

$$
\mathrm{IC}=\frac{40000 . \Pi \cdot \mathrm{A}}{\mathrm{P}^{2}}
$$

em que IC = índice de circularidade; $\mathrm{A}=$ área do fragmento, em ha; e $\mathrm{P}=$ perímetro do fragmento, em $\mathrm{m}$.

O fragmento apresentará tendência à forma arredondada, quando o valor do índice de circularidade (IC) for próximo de 1. À medida que este se distancia de 1 , tem-se um fragmento alongado.

\section{RESULTADOS E DISCUSSÃO}

Nas análises realizadas no Mapa da Carta do IBGEMCI e no Mapa dos Fragmentos Florestais do Município de Jaboticaba-MFJ, foram observados 122 e 121 fragmentos florestais, com área total de 3.770,70 ha e 1.038,6 ha, e perímetro total de 246.308,90 me 152.996,98 $\mathrm{m}$, respectivamente (Figuras 1 e 2 e Tabelas 1 e 2).

Apesar de apresentar, praticamente, o mesmo número de fragmentos florestais, observou-se diminuição considerável na área de mata no decorrer desses 29 anos.

No fragmento 104 do Mapa da Carta do IBGEMCI (Figura 1), denotou-se que, de uma área de 788,40 ha, restaram apenas dois fragmentos 97 e 98 Mapa dos Fragmentos Florestais do Município de JaboticabaMFJ (Figura 2), com área total de 13,82 ha, isto é, apenas $1,75 \%$ restante do fragmento florestal anterior.

De acordo com os dados do IBGE, a área rural do município é de 66.940 ha. Considerando os fragmentos florestais mapeados no $\boldsymbol{M C I}$ (Figura 1), observou-se uma ocupação de $3.770,70$ ha, ou seja, 3,63\% da área com vegetação natural (Tabela 1). Entretanto, em 2000 o cenário foi muito diferente, restando apenas $1,55 \%$ da vegetação natural, isto é, 1.038,26 ha (Tabela 2). Assim, denotou-se que o município estava aquém da área natural exigida pelas leis ambientais.

Oliveira et al. (2005), em estudos do volume de povoamento para fragmentos florestais naturais do Município de Viçosa, Minas Gerais, observaram a presença de 289 fragmentos, recobrindo $22,77 \%$ da área do município. Apesar de apresentarem essa porcentagem, os autores relataram que a maioria dos fragmentos estava pequena, podendo estar sob efeito de borda.

No $\boldsymbol{M C I}$, observou-se apenas 24 fragmentos (19,67\%) menores que 5 ha, representando 2,02\% (76,28 ha) da área total. Já no $\boldsymbol{M F J}$ esse número aumentou para $69(57,02 \%)$, representando $13,60 \%$ (141,25 ha). Entre as áreas com 80 a 200 ha no $\boldsymbol{M C I}$, verificouse que existiam seis fragmentos $(4,92 \%)$, entretanto no $\boldsymbol{M F} \boldsymbol{J}$ se observou apenas um fragmento $(0,83 \%)$. Os fragmentos maiores que 200 ha não foram mais observados no $\boldsymbol{M F J}$, comprovando a diminuição considerável dessas áreas e a fragmentação das matas no município (Figuras 1 e 2 e Tabelas 1 e 2).

O menor fragmento encontrado no $\boldsymbol{M F J}$ foi o 31 , com 0,36 ha e perímetro de 294,66 m. De acordo com Sanders et al. (1991), remanescentes de área pequena apresentam frágeis padrões de sustentabilidade ao longo do tempo e, segundo Bierregaard Junior et al. (1992), estes não têm capacidade de proteger a diversidade biológica. Com base nessas informações, é preciso levar em consideração a possibilidade de que o fragmento 31 seja eliminado da paisagem, caso não sejam adotadas propostas de manejo que promovam aumento de sua área e interligação com fragmentos próximos e maiores.

R. Árvore, Viçosa-MG, v.33, n.1, p.117-124, 2009 


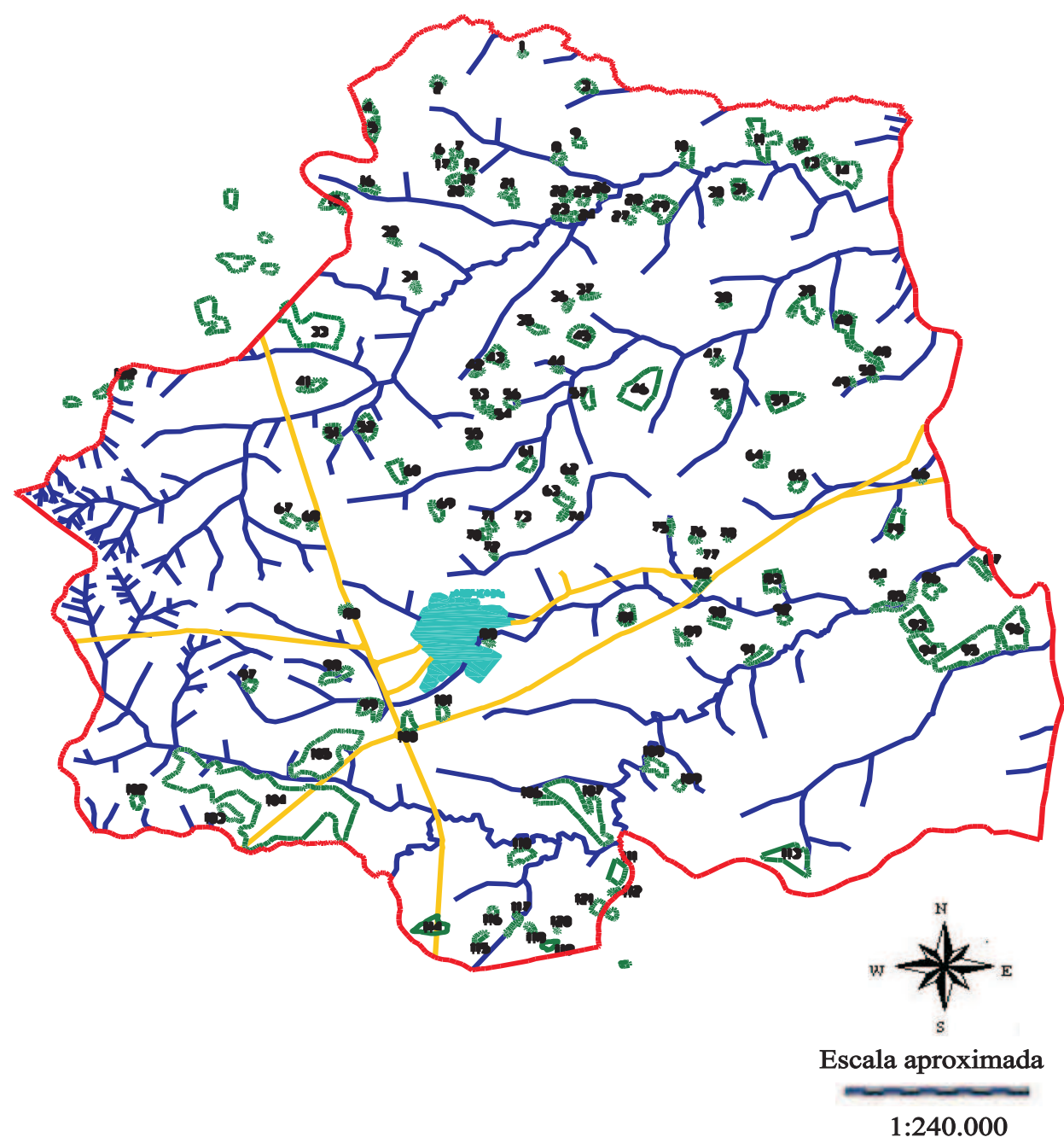

Figura 1 - Mapa da Carta do IBGE-MCI dos fragmentos florestais de 1971 do Município de Jaboticabal, Estado de São Paulo.

Figure 1 -IBGE-MCI map of the 1971 forest fragments inJaboticabal-SP.

Tabela 1 - Classes de área dos fragmentos florestais do Município de Jaboticabal, Estado de São Paulo Table 1 - Forest fragment area classes in Jaboticabal-SP

\begin{tabular}{|c|c|c|c|c|c|c|c|c|c|c|}
\hline \multirow[t]{4}{*}{ Classes de Área (ha) } & \multicolumn{10}{|c|}{ Fragmentos Florestais } \\
\hline & \multicolumn{5}{|c|}{1971} & \multicolumn{5}{|c|}{2000} \\
\hline & \multicolumn{2}{|c|}{ Número de ocorrência } & \multicolumn{3}{|c|}{ Área } & \multicolumn{2}{|c|}{ Número de ocorrência } & \multicolumn{3}{|c|}{ Área } \\
\hline & Absoluto & $\%$ & ha & $\%$ & Média & Absoluto & $\%$ & ha & $\%$ & Média \\
\hline$<5,0$ & 24 & 19,67 & 76,28 & 2,02 & 3,18 & 69 & $57,02 \%$ & 141,25 & 13,60 & 2,05 \\
\hline $5,0--10,0$ & 33 & 27,05 & 243,85 & 6,47 & 7,39 & 26 & $21,49 \%$ & 199,70 & 19,23 & 7,68 \\
\hline $10,0--20,0$ & 28 & 22,95 & 425,39 & 11,28 & 15,19 & 13 & $10,74 \%$ & 163,87 & 15,78 & 12,61 \\
\hline $20,0--80,0$ & 30 & 24,59 & 1156,42 & 30,67 & 38,55 & 12 & $9,92 \%$ & 420,92 & 40,54 & 35,08 \\
\hline $80,0--\mid 200,0$ & 6 & 4,92 & 1004,08 & 26,63 & 167,35 & 1 & $0,83 \%$ & 112,52 & 10,84 & 112,52 \\
\hline$>200,0$ & 1 & 0,82 & 788,40 & 20,91 & 788,40 & 0 & $0 \%$ & 0 & 0 & 0 \\
\hline Total & 122 & 100 & 3770,70 & 100 & 30,91 & 121 & $100 \%$ & 1038,26 & 100 & 8,58 \\
\hline
\end{tabular}

R. Árvore, Viçosa-MG, v.33, n.1, p.117-124, 2009 


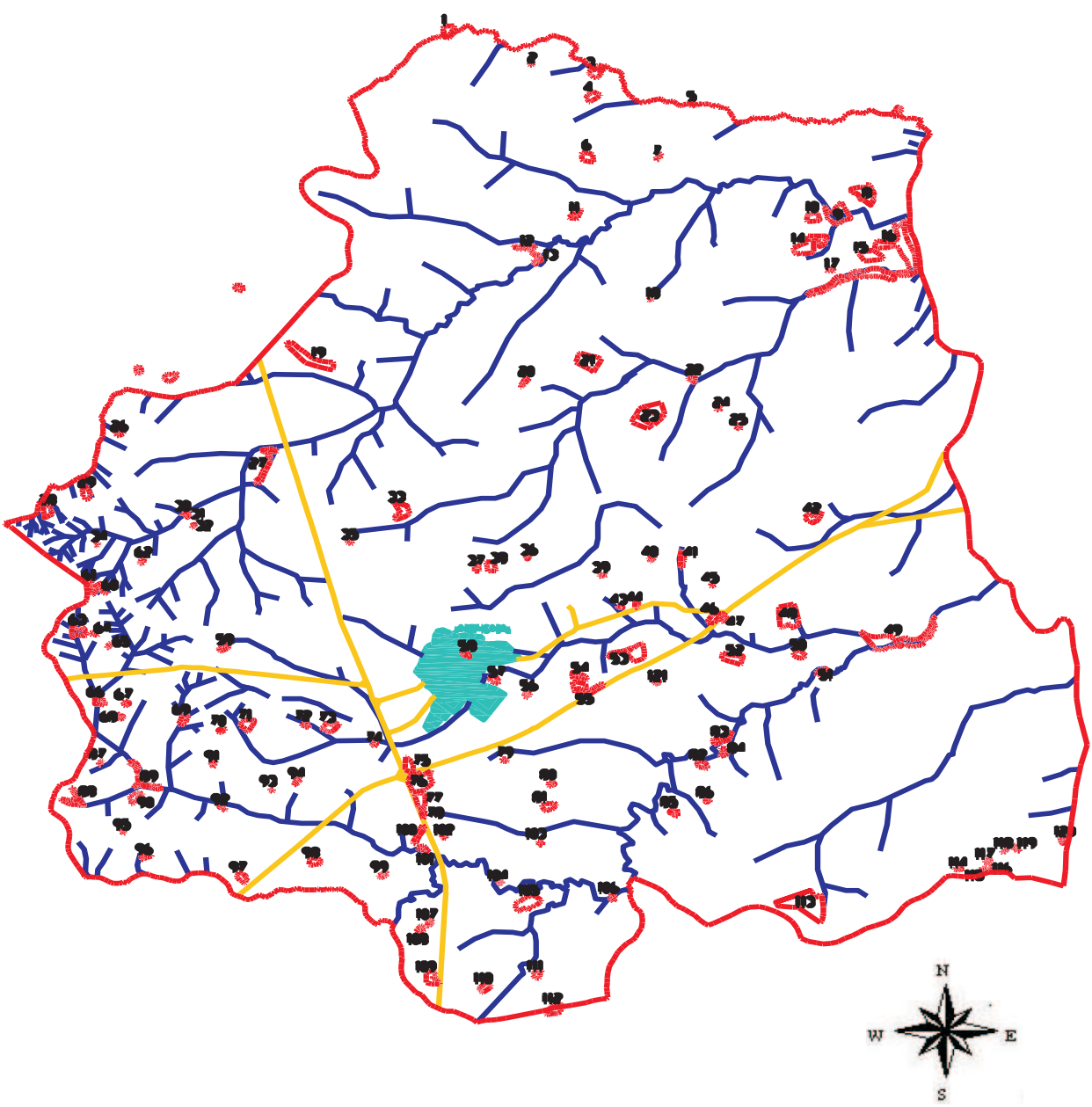

Escala aproximada

$1: 240.000$

Figura 2 - Mapa dos fragmentos florestais de 2000 do Município de Jaboticabal-MFJ, Estado de São Paulo. Figure 2 - Map of the 2000 forest fragments in Jaboticabal-SP.

Tabela 2 - Classes de perímetro dos fragmentos florestais do Município de Jaboticabal, Estado de São Paulo Table 2 - Forest Fragment perimeter classes in Jaboticabal-SP

\begin{tabular}{|c|c|c|c|c|c|c|c|c|c|c|}
\hline \multirow{4}{*}{$\begin{array}{l}\text { Classes de } \\
\text { Perimetro (m) }\end{array}$} & \multicolumn{10}{|c|}{ Fragmentos Florestais } \\
\hline & & & \multicolumn{3}{|c|}{$\begin{array}{l}1971 \\
\text { Área }\end{array}$} & & & \multicolumn{3}{|c|}{2000} \\
\hline & \multicolumn{2}{|c|}{ Número de ocorrência } & & Área & & \multicolumn{2}{|c|}{ Número de ocorrência } & & Área & \\
\hline & Absoluto & $\%$ & $\mathrm{~m}$ & $\%$ & Média & Absoluto & $\%$ & $\mathrm{~m}$ & $\%$ & Média \\
\hline$<1000$ & 25 & 20,49 & 18439,60 & 7,49 & 737,58 & 70 & 57,85 & 42475,54 & 27,76 & 606,79 \\
\hline $1000--1500$ & 36 & 29,51 & 43455,30 & 17,64 & 1207,09 & 23 & 19,01 & 27791,93 & 18,17 & 1208,34 \\
\hline 2000 & 21 & 17,21 & 36426,50 & 14,49 & 1734,60 & 12 & 9,92 & 19882,51 & 13,00 & 1656,88 \\
\hline 2500 & 15 & 12,30 & 33524,90 & 13,61 & 2234,99 & 3 & 2,48 & 6662,34 & 4,85 & 2220,78 \\
\hline $2500--3500$ & 12 & 9,84 & 33682,40 & 13,67 & 2806,87 & 8 & 6,61 & 24171,40 & 15,80 & 3021,43 \\
\hline $3500--5500$ & 8 & 6,56 & 33667,00 & 13,67 & 4208,38 & 3 & 2,48 & 11580,52 & 7,57 & 3860,17 \\
\hline $5500--50000$ & 5 & 4,10 & 47113,20 & 19,13 & 9422,64 & 2 & 1,65 & 20432,74 & 13,35 & 10216,37 \\
\hline Total 122 & 100 & 246308,90 & 100 & 2018,93 & 121 & $100 \quad 1$ & 152996,98 & 100 & 1264,44 & \\
\hline
\end{tabular}


Foi observado que recentemente apenas 12 fragmentos possuíam em média 35,08 ha, ocupando $40,54 \%$ da área total relativa aos fragmentos mapeados, ou seja, 420,92 ha, em comparação com 1971, em que havia 30 fragmentos com média de 30,55 ha ocupando $30,67 \%$ da área total de fragmentos (Tabela 1), totalizando esses $1.156,42$ ha de matas no município. O maior fragmento encontrado em 1971 foi o fragmento 104 (Figura 1) e em 2000, o fragmento 16 (Figura 2), possuindo, respectivamente, 788,40 ha $112,52 \mathrm{ha}$. O primeiro ocupava $20,91 \%$ da área total de fragmentos mapeados e o segundo, $10,84 \%$ da área.

Os fragmentos de maiores perímetros (5.500-50.000 $\mathrm{m})$ apresentaram valores totais em $1971 \mathrm{de} 47.113,20 \mathrm{~m}$, ocupando 19,13\% da área total. Em 2000, apresentaram valores totais de $20.432,74 \mathrm{~m}$, com $13,35 \%$ da área total (Tabela 2). O total do perímetro mapeado dos fragmentos foi de $246.308,90 \mathrm{~m}$ e $152.9926,98 \mathrm{~m}$, respectivamente, em 1971 e 2000, possuindo em média 2.018,93 me 1.264,44 m. Contudo, observa-se ainda, na Tabela 2, que em 1971, dos 122 fragmentos florestais mapeados, $50 \%$ possuíam perímetro inferior a $1.500 \mathrm{~m}$. Em 2000, dos 121 fragmentos, $76,86 \%$ apresentavam perímetro inferior a $1.500 \mathrm{~m}$. Denotouse, portanto, aumento considerável de fragmentos menores na evolução temporal do município.

\section{Índice de Circularidade ( IC )}

A forma dos fragmentos foi determinada na análise do Índice de Circularidade (IC), ou relação borda/interior (Tabela 3).

A importância dessa análise para os estudos da dinâmica e estrutura dos fragmentos florestais evidenciase na possibilidade de indicar o nível de proteção de seu interior em relação aos efeitos de borda. Viana e Pinheiro (1998) estudaram a forma de fragmentos florestais na região de Piracicaba, utilizando o parâmetro fator de forma, que é a relação entre a área do fragmento e seu perímetro, e a classificação determinada foi a seguinte: fragmentos com fator de forma superior a 0,8 são considerados arredondados; entre 0,6 e 0,8 são alongados; e inferior a 0,6, muito alongados.

Na análise da Tabela 3, em 1971 e em 2000, 15 e 17 fragmentos, respectivamente, apresentavam índice de circularidade acima de 0,8 . Observa-se, nessa tabela, que nas duas épocas a maior parte dos fragmentos mapeados apresentou formas moderadamente alongadas entre 0,6 e 0,8, 32 e 31 em 1971; e 23 e 36 em 2000. As formas muito alongadas, isto é, menores que 0,6 , foram observadas em 44 fragmentos em 1971 e 45 em 2000.
Tabela 3 - Classes de índice de circularidade dos fragmentos florestais do Município de Jaboticabal, Estado de São Paulo

Table 3 - Forest fragment circle index classes in Jaboticabal-SP

\begin{tabular}{|c|c|c|c|c|}
\hline \multirow[t]{3}{*}{$\overline{\text { Classes Ic }}$} & \multicolumn{4}{|c|}{ Fragmentos Florestais } \\
\hline & \multicolumn{2}{|c|}{1971} & \multicolumn{2}{|c|}{2000} \\
\hline & $\begin{array}{l}\text { Número de } \\
\text { Ocorrência }\end{array}$ & $\%$ & $\begin{array}{l}\text { Número de } \\
\text { Ocorrência }\end{array}$ & $\%$ \\
\hline$<0,170$ & 1 & $0,82 \%$ & 4 & $3,31 \%$ \\
\hline $0,170--0,500$ & 18 & $14,75 \%$ & 21 & $17,35 \%$ \\
\hline $0,500--0,600$ & 25 & $20,49 \%$ & 20 & $16,52 \%$ \\
\hline $0,600--0,700$ & 32 & $26,23 \%$ & 23 & $19,01 \%$ \\
\hline $0,700--0,800$ & 31 & $25,41 \%$ & 36 & $29,75 \%$ \\
\hline $0,800--1,000$ & 15 & $12,29 \%$ & 17 & $14,05 \%$ \\
\hline Total & 122 & $100 \%$ & 121 & $100 \%$ \\
\hline
\end{tabular}

Nascimento et al. (2006) desenvolveram um estudo do uso da terra e diagnosticaram os fragmentos de vegetação florestal nativa, na bacia hidrográfica do Rio Alegre no Estado do Espírito Santo. Esses autores concluíram que a maioria dos fragmentos florestais possuía forma fortemente alongada e a área média de 6,3 ha, com a maior parte sujeita a um elevado nível de perturbação, tendo em vista a cultura cafeeira na região.

Na fragmentação da floresta ocorrem mudanças imediatas e pronunciadas, iniciando-se pelas maiores luminosidade, temperatura, umidade e temperatura do vento. Essas mudanças são mais pronunciadas na borda e diminuem na direção do interior da floresta. Nas plantas, os efeitos de borda podem ser diretos (ex. climáticos) ou indiretos (ex. interações com polinizadores, dispersores, cipós etc.). Por exemplo, com o aumento da luminosidade na borda dos fragmentos ocorre aumento no crescimento de espécies pioneiras invasoras, especialmente cipós (LEWIN, 1984).

Apesar de o Mapa dos Fragmentos Florestais de 2000 (Figura 2) apresentar um número maior de fragmentos com índice de circularidade próximo do ideal, os fragmentos apresentaram áreas menores que os mesmos mapeados em 1971. André et al. (1997) afirmaram que o efeito de borda chega ao redor de 80-100 m, adentrando a mata; e Lovejoy et al. (1986) declararam que fragmentos de até 10 ha são totalmente afetados por esse efeito. Portanto, de acordo com a Tabela 1, observa-se que, em 2000, 78,51\% dos fragmentos mapeados apresentam áreas menores que 10 ha, e na Tabela 3 denota-se que em 2000, mesmo apresentando 17 fragmentos com índice de circularidade ideal, estes estão sob o efeito de borda total. 
Enfim, a situação da vegetação natural do município encontra-se fortemente fragmentada. Essa observação vai de encontro com o que se observou no Estado de São Paulo, que possui paisagens fortemente alteradas pela ocupação antrópica, apresentando apenas cerca de $12 \%$ de cobertura florestal nativa remanescente (SÃO PAULO, 1993). A recuperação destas áreas degradadas se configura em uma das principais alternativas para, entre outros aspectos, diminuir o risco de perda de espécies e ecossistemas ameaçados de extinção e resgatar importantes funções perdidas durante o processo de degradação da cobertura florestal (BARBOSA e MANTOVANI, 2000; LIMA e ZAKIA, 2000).

\section{CONCLUSÕES}

As áreas de remanescentes de fragmentos florestais do Município de Jaboticabal, SP, com a evolução temporal, entre 1971 e 2000, diminuíram, mas o número de fragmentos não.

Os fragmentos florestais de maior área, observados em 1971, apresentaram áreas extremamente menores em 2000.

Os fragmentos florestais tiveram, em 2000, índice de circularidade ideal, com efeito de borda acentuado.

\section{AGRADECIMENTOS}

Ao técnico agrícola Ronaldo José de Barros, pela digitalização; à desenhista Izilda M.C. Máxim, pelo desenvolvimento do mapa; e à UNESP/FCAV, pelo apoio a esta pesquisa.

\section{REFERÊNCIAS}

ANDRÉ, A. J. et al. Conseqüências da fragmentação e do efeito de borda sobre a estrutura, diversidade e sustentabilidade de um fragmento de floresta de planalto de Piracicaba, SP. Revista Brasileira de Biologia, v.57, n.1, p.47-60, 1997.

APPOLINÁRIO, V.; OLIVEIRA-FILHO, A. T.; GUILHERME, F. A. G. Tree population and community dynamics in a Brazilian tropical semideciduous forest. Revista Brasileira de Botânica, v.28, n.2, p.347-360, 2005.
BARBOSA, L. M.; MANTOVANI, W. Degradação ambiental: conceituação e bases para o repovoamento vegetal. In: BARBOSA, L. M. (Coord.) WORKSHOP SOBRE RECUEPRAÇÃO DE ÁREAS DEGRADADAS DA SERRA DO MAR E FORMAÇÕES FLORESTAIS LITORÂNES, 2000, São Paulo. Anais... São Paulo: Secretaria do Meio Ambiente, 2000. p.34-40.

BIERREGAARD JÚNIOR, R. et al. The biological dynamics of troical rainflorest fragments a prospective comparision of fragment and continuous forest. Bioscience, v.42, n.11, p.859-866, 1992.

INSTITUTO BRASILEIRO DE GEOGRAFIA IBGE/Ministério do Planejamento e coordenação Geral Fundação. Carta do Brasil. Folha SF22-X-III-3. Escala 1:50.000.

INSTITUTO BRASILEIRO DE GEOGRAFIA IBGE Censo 2000. Disponível em: $<$ http:// www.ibge.gov.br/censo/>. Acesso em 10 de dez. 2006

Jaboticabal 2006. Disponível em: http:// www.jaboticabal.sp.gov.br/jaboticabal/. Acesso em 8 de dezembro de 2006.

KORNING, J.; BALSLEV, H. Growth and mortality of trees in Amazonian tropical rain forest in Ecuador. Journal of Vegetation Science, v.4,n.1, p.77-86, 1994.

LEWIN, R. Parks: how big is big enough? Science, v.225, p.611-612, 1984.

LIMA, W. P.; ZAKIA, M. J. B. Hidrologia de matas ciliares. In: RODRIGUES, R. R.; LEITÃO FILHO, H. F. Matas ciliares: conservação e recuperação. São Paulo: EDUSP, 2000. p.33-44.

LOVEJOY, T. E. et al. Edge and other effects of isolation on Amazon forest fragments. In: SOULÉ, M.E., Ed.. Conservation biology.

Sunderland: Sinauer Press, 1986. p.257-285,

NASCIMENTO, M. C. et al. Mapeamento dos fragmentos de vegetação florestal nativa da Bacia hidrográfica do rio Alegre, Espiroto Santo, a partir de imagens do Satélite IKONOS II. Revista Árvore, v.30, n.3, p.389-398, 2006.

R. Árvore, Viçosa-MG, v.33, n.1, p.117-124, 2009 
OLIVEIRA, M. L. R. et al. Equações de volume de povoamento para fragmentos florestais naturais do município de Viçosa, Minas Gerais. Revista Árvore, v.29, n.2, p.213-225, 2005.

PISSARRA, T. C. T. Análise da bacia hidrográfica do Córrego Rico na subregião de Jaboticabal, SP: comparação entre imagens TM-Landsat 5 e fotografias aéreas verticais. 2002. 136f. Tese (Doutorado em Agronomia - Área de Concentração em Produção Vegetal) Universidade Estadual Paulista, Jaboticabal, 2002.

PULLIAM, H. R. Sources and sinks: empirical evidence and population consequences. In: RHODES, O. E.; CHESSER, R. K.; SMITH, M. H. (Eds.). Population dynamics in space and time. Chicago: The University of Chicago Press, 1996. p.45-70.
SÃO PAULO (ESTADO). Secretaria do Meio Ambiente. Instituto Florestal. Inventário florestal do estado de São Paulo. São Paulo: 1993. 199p.

SAUNDERS, D. A.; HOBBS, R. J.; MARGULES, C. $\mathrm{R}$. Biological consequences of ecosystem fragmentation: a review. Conservation Biology, v.5, n.1, p.18-34, 1991.

VIANA, V. M. Biologia e manejo de fragmentos florestais naturais. In: CONGRESSO FLORESTAL BRASILEIRO, 6., 1990, Campos do Jordão. Anais... Campos do Jordão: SBS/SBEF, 1990. p.113-118.

VIANA, V. M.; PINHEIRO, L. A. F. V. Conservação da biodiversidade em fragmentos florestais. IPEF, v. 12, n.32, p. 25-42, 1998. 